\title{
Identification of Genetic Causes of Inherited Peripheral Neuropathies by Targeted Gene Panel Sequencing
}

\author{
Soo Hyun Nam', Young Bin Hong', Young Se Hyun', Da Eun Nam', Geon Kwak ${ }^{3,4}$, Sun Hee Hwang ${ }^{3}$, \\ Byung-Ok Cho ${ }^{2,3,4}$, and Ki Wha Chung ${ }^{1, *}$
}

\begin{abstract}
Inherited peripheral neuropathies (IPN), which are a group of clinically and genetically heterogeneous peripheral nerve disorders including Charcot-Marie-Tooth disease (CMT), exhibit progressive degeneration of muscles in the extremities and loss of sensory function. Over 70 genes have been reported as genetic causatives and the number is still growing. We prepared a targeted gene panel for IPN diagnosis based on next generation sequencing (NGS). The gene panel was designed to detect mutations in 73 genes reported to be genetic causes of IPN or related peripheral neuropathies, and to detect duplication of the chromosome $17 p 12$ region, the major genetic cause of CMT1A. We applied the gene panel to 115 samples from 63 non-CMT1A families, and isolated 15 pathogenic or likelypathogenic mutations in eight genes from 25 patients (17 families). Of them, eight mutations were unreported variants. Of particular interest, this study revealed several very rare mutations in the SPTLC2, DCTN1, and MARS genes. In addition, the effectiveness of the detection of CMT1A was confirmed by comparing five 17p12-nonduplicated controls and 15 CMT1A cases. In conclusion, we developed a gene panel for one step genetic diagnosis of IPN. It seems that its time- and cost-effectiveness are superior to previous tiered-genetic diagnosis algorithms, and it could be applied as a genetic diagnostic system for inherited peripheral neuropathies.
\end{abstract}

\footnotetext{
${ }^{1}$ Department of Biological Sciences, Kongju National University, Gongju 32588, Korea, ${ }^{2}$ Stem Cell \& Regenerative Medicine Center and Neuroscience Center, Samsung Medical Center, Seoul 06351, Korea, ${ }^{3}$ Department of Neurology, Sungkyunkwan University School of Medicine, Seoul 06351, Korea, ${ }^{4}$ Department of Health Sciences and Technology, Samsung Advanced Institute for Health Science \& Tech., Sungkyunkwan University, Seoul 06351, Korea

${ }^{*}$ Correspondence: kwchung @kongju.ac.kr (KWC); bochoi77@ hanmail.net (BOC)
}

Received 20 October, 2015; revised 11 February, 2016; accepted 22 February, 2016; published online 30 March, 2016

Keywords: charcot-Marie-Tooth disease (CMT), DCTN1, gene panel, inherited peripheral neuropathy, MARS, SPTLC2

\section{INTRODUCTION}

Inherited peripheral neuropathies (IPNs) are a group of disorders comprising clinically heterogeneous diseases. These include hereditary motor and sensory neuropathy (usually called Charcot-Marie-Tooth disease: CMT), distal hereditary motor neuropathy (dHMN), hereditary sensory autonomous neuropathy (HSAN), hereditary neuropathy with a liability to pressure palsy (HNPP), and congenital hypomyelinating neuropathy (CHN) (Lupski and Garcia, 2000; Patzkó and Shy, 2011). IPNs are characterized by progressive distal motor weakness, sensory loss, and areflexia in the upper and lower limbs. Genetically, IPNs are very heterogeneous and over 70 related genes have been filed (Rossor et al., 2013; Rudnik-Schöneborn et al., 2016). They have shown the loose genotype-phenotype correlation and phenotypic overlapping between the disease types (Gonzaga-Jauregui et al., 2015; Nakhro et al., 2013). Mutations in a specific gene frequently cause different types of IPNs, whereas similar phenotypes are usually caused by different genetic defects. As an example, mutations in the MPZ gene (MIM 159440) are known to cause several IPN types including dominant CMT1B (MIM 118200), CMT2I (MIM 607677), CMT2J (MIM 607736), DSS (MIM 145900), DI-CMTD (MIM 607791), and recessive CHN (CMT4E, MIM 605253). This clinical and genetic heterogeneity makes their exact diagnosis difficult.

Although the genetic diagnosis of IPNs has been conventionally dependent on the direct sequencing of major causative genes, it has low isolation efficiency in spite of the laborious procedure. The recent advent of whole exome sequencing (WES) based on the next-generation sequencing (NGS) technique has broadened and accelerated the analysis of various disorders at the level of genome especially in heterogeneous disorder groups such as IPNs (Choi et al., 2012; Drew et al., 2015; Gonzaga-Jauregui et al., 2015)., WES is particularly efficient for identifying rare genetic causes from wide-ranging causative genes. Application of WES to CMT has several additional benefits. WES is cost- and time-effective compared to previous methods or procedures. In addition, advances in detection algorithms for single nucleotide variation (SNV), has increased their accuracy. From the first application in 2011 (Montenegro et al., 2011), NGS has been applied in more than hundreds studies to isolate underlying causes of IPN. More recently, targeted multi-gene panel sequencing has been applied as an alternative method for genetic testing of several

elSSN: 0219-1032

(c) The Korean Society for Molecular and Cellular Biology. All rights reserved.

(c) This is an open-access article distributed under the terms of the Creative Commons Attribution-NonCommercial-ShareAlike 3.0 Unported License. To view a copy of this license, visit http://creativecommons.org/licenses/by-nc-sa/3.0/. 
Table 1. Inherited peripheral neuropathy families involved in target sequencing

\begin{tabular}{|c|c|c|c|c|c|}
\hline \multirow{2}{*}{ Type $^{a}$} & \multirow{2}{*}{ Family number } & \multicolumn{3}{|c|}{ Subject number } & \multirow{2}{*}{ Genetically diagnosed family } \\
\hline & & Affected & Unaffected & Total & \\
\hline CMT1 & 16 & 17 & 6 & 23 & 1 \\
\hline СMT2 & 30 & 32 & 23 & 55 & 4 \\
\hline CMT4 & 1 & 1 & 3 & 4 & 1 \\
\hline CMTX & 12 & 19 & 8 & 27 & 9 \\
\hline $\mathrm{dHMN}$ & 2 & 2 & 1 & 3 & 1 \\
\hline HSAN & 2 & 3 & 0 & 3 & 1 \\
\hline CMT1A & 15 & 15 & 5 & 20 & - \\
\hline Total & 78 & 89 & 46 & 135 & 17 \\
\hline
\end{tabular}

${ }^{a}$ CMT1, Charcot-Marie-Tooth disease type 1 with autosomal dominant demyelination; CMT2, CMT type 2 with axonal defect; CMT4, CMT type 4 with autosomal dominant demyelination; dHMN, distal hereditary motor neuropathy; HSAN, hereditary sensory autonomous neuropathy; CMT1A, CMT type $1 \mathrm{~A}$ with $17 \mathrm{p} 12$ duplication.

neuropathic disorders (Antoniadi et al., 2015; Ylikallio et al., 2014). Multi-gene panel testing is particularly effective for the diagnosis of neurodegenerative disorders with large clinical and pathogenic spectra.

Here, we designed and validated a new targeted gene panel for one step, simultaneous genetic diagnosis of IPNs by detection of $17 \mathrm{p} 12$ duplication and SNVs in subject of 78 Korean IPN families.

\section{MATERIALS AND METHODS}

\section{Subjects}

This study enrolled a total of 78 Korean IPN families (89 affected and 46 unaffected individuals) with variable subtypes, such as CMT1, CMT2, intermediate CMT (IntCMT), dHMN, and HSAN (Table 1). Of the IPN families, 15 CMT1A (MIM 118220) families who were previously diagnosed to have $17 \mathrm{p} 12$ (PMP22) duplication were included. In addition, 300 healthy Korean controls were included in this study. All participants provided written informed consents according to the protocol approved by the Institutional Review Board for Sungkyunkwan University, Samsung Medical Center (\#SMC2013-10-066).

\section{Clinical assessments}

Clinical information included motor and sensory impairments, deep tendon reflexes, and muscle atrophy. Age at onset was determined by asking patients for their ages, when symptoms (i.e., distal muscle weakness, foot deformity, or sensory change) first appeared. Physical disability was measured by the functional disability scale (FDS) and the CMT neuropathy score (CMTNS). Nerve conduction studies including motor and sensory conduction velocities were measured on the median, ulnar, peroneal, tibial, and sural nerves.

\section{DNA purification and prescreening of 17p12 duplication}

Genomic DNA was purified from peripheral blood using a QIAamp blood DNA purification kit (Qiagen, Germany). All patient family samples were prescreened for the 17p12 (PMP22) duplication, which is the most frequent genetic cause of demyelinating CMT, using a hexaplex microsatellite PCR (Choi et al., 2007).

Design and preparation of gene panel

A total of 73 target genes were selected by extensive search of literatures and databases (Supplementary Table S1). A total of 1669 regions were read by 6298 probes with coverage of $99.5 \%$. The probe design was performed according to Suredesign website (https://earray.chem.agilent.com/suredesign), and then DNA samples were prepared using the SureSelect DNA Sample Preparation System (Agilent Technologies, USA). Quality control and quantification of the prepared libraries were performed by Macrogen, Inc. (Korea).

\section{Sequencing and identification of causative mutations} Sequencing was performed using a HiSeq 2000 Genome Analyzer (Illumina, USA) and the UCSC assembly hg19 (GRCh37) was used as the reference sequence(http://genome.ucsc.edu). Functionally significant variants (missense, nonsense, exonic insertion/deletion (indel), and splicing site variants) were selected, and then novel or uncommon variants (minor allele frequency $\leq 0.01$ ) were further selected by searching the globally available human genome variant databases, such as dbSNP144 databases (http://www.ncbi.nlm.nih.gov), the 1000 Genomes Project (1000G) (http://www.1000genomes.org/), Exome Variant Project (ESP) (http://evs.gs.washington.edu/EVS/), and the Exome Aggregation Consortium (ExAC) (http://exac.broadinstitute.org/). The Sanger's sequencing method was applied to confirm candidate variants in the extended family members using a genetic analyzer ABI3130XL (Life Technologies, USA). For the finally selected pathogenic or likely pathogenic variants, the genomic evolutionary rate profiling (GERP) scores were determined by the GERP++ program (http://mendel.stanford. edu/SidowLab/downloads/gerp/index.html). In silico analyses of the mutant proteins were performed using the prediction algorithms of SIFT (http://sift.jcvi.org), MUpro (http://www.ics.uci. edu/ baldig/mutation), and PolyPhen-2 (http://genetics.bwh. harvard.edu/pph2/).

\section{RESULTS}

General properties of the targeted-gene diagnostic panel The gene panel included 73 IPN-relevant genes and one control gene with a target size of $276 \mathrm{~kb}$ (Supplementary Table S1). We performed genetic tests for 74 genetically un-diagnosed CMT patients who had been excluded 17p12 (PMP22) duplication (Table 1). Mean read depth was 194.8X, and target coverage with read depth of $\geq 10 \mathrm{X}$ was $98.9 \%$ (Supplementary Table S2). Total numbers of SNVs (including SNPs and indels) 
Gene Panel for Molecular Diagnosis of IPN

Soo Hyun Nam et al.

Table 2. Summary of pathogenic or likely pathogenic mutations from the gene panel sequencing

\begin{tabular}{|c|c|c|c|c|c|c|c|c|c|}
\hline \multirow{2}{*}{ Type } & \multirow{2}{*}{ Gene } & \multicolumn{2}{|c|}{ Mutation } & \multirow{2}{*}{ Family } & \multirow{2}{*}{$\begin{array}{l}\text { Inheriting } \\
\text { mode }\end{array}$} & \multirow{2}{*}{$\begin{array}{c}\text { Onset } \\
\text { (yrs) }\end{array}$} & \multicolumn{2}{|c|}{ Severity ${ }^{b}$} & \multirow{2}{*}{ References } \\
\hline & & $\mathrm{Nt}$ & $\mathrm{AA}^{\mathrm{a}}$ & & & & CMTNS & FDS & \\
\hline CMT1E & PMP22 & c. $47 \mathrm{~T}>\mathrm{C}$ & p.L16R ${ }^{\star}$ & FC541 & $\begin{array}{c}\mathrm{AD} \\
\text { de novo }\end{array}$ & 1 & 24 & 6 & This study \\
\hline CMT4C & SH3TC2 & $\begin{array}{l}\text { c. } 929 \mathrm{G}>\mathrm{A}+ \\
\text { c. } 3272 \mathrm{G}>\mathrm{T}\end{array}$ & $\begin{array}{l}\text { p.G310E* }{ }^{*} \\
\text { p.G1091V* }\end{array}$ & FC703 & $\mathrm{AR}$ & 5 & 15 & 3 & This study \\
\hline CMT2A & MFN2 & $\mathrm{c} .839 \mathrm{G}>\mathrm{A}$ & p.R280H & FC527 & $A D$ & 45 & 10 & 3 & Chung et al., 2006 \\
\hline CMT2I & $M P Z$ & $\begin{array}{l}\text { c. } 154 T>G \\
\text { c. } 262 T>C\end{array}$ & $\begin{array}{l}\text { p.F52V* } \\
\text { p.Y88H* }\end{array}$ & $\begin{array}{l}\text { FC156 } \\
\text { FC141 }\end{array}$ & $\begin{array}{c}A D \\
A D \\
\text { de novo }\end{array}$ & $\begin{array}{l}25 \\
20\end{array}$ & $\begin{array}{l}16 \\
17\end{array}$ & $\begin{array}{l}3 \\
3\end{array}$ & $\begin{array}{l}\text { This study } \\
\text { This study }\end{array}$ \\
\hline CMT2U & MARS & c. $2398 \mathrm{C}>\mathrm{A}$ & p.P800T & FC495 & $A D$ & 5 & 5 & 1 & Hyun et al., 2014 \\
\hline \multirow[t]{9}{*}{ CMTX1 } & GJB1 & $\mathrm{c} .20 \mathrm{~A}>\mathrm{G}$ & p.Y7C & FC718 & $X D$ & 15 & 12 & 2 & Schiavon et al., 1996 \\
\hline & & c. $43 \mathrm{C}>\mathrm{T}$ & p.R15W & FC751 & $X D$ & 49 & 5 & 1 & Nelis et al., 1996 \\
\hline & & c. $283 G>A$ & p.V95M & FC565 & $X D$ & 48 & 9 & 2 & Bone et al., 1997 \\
\hline & & & & FC687 & $X D$ & 8 & 4 & 1 & \\
\hline & & & & FC698 & $X D$ & 40 & 11 & 2 & \\
\hline & & c. $286 G>C$ & p.A96P* & FC725 & $X D$ & 11 & 12 & 2 & This study \\
\hline & & c. $490 \mathrm{C}>\mathrm{T}$ & p.R164W & FC714 & $X D$ & 30 & 15 & 4 & Oterino et al., 1996 \\
\hline & & c. $491 \mathrm{G}>\mathrm{A}$ & p.R164Q & FC254 & $X D$ & 15 & 11 & 2 & Bone et al., 1997 \\
\hline & & & & FC722 & $X D$ & 8 & 20 & 3 & \\
\hline HSAN1C & SPTLC2 & $c .435 G>T$ & p.R145S* & FC459 & $A D$ & 17 & 15 & 3 & This study \\
\hline dHMN7B & DCTN1 & c. $1019 A$ > G & p.E340G* & FC180 & $A D$ & 10 & 12 & 3 & This study \\
\hline
\end{tabular}

AA, amino acid; AD, autosomal dominant; AR, autosomal recessive; CMTNS, Charcot-Marie-Tooth disease neuropathy score; FDS, functional disability scale; Nt, nucleotide; XD, X-linked dominant

${ }^{\mathrm{a}}$ Mutations with *: likely pathogenic

${ }^{\mathrm{b}}$ CMTNS: mild: $\leq 10$, moderate: 11-20, and severe: $\geq 21$; FDS: 0 : normal, 1: normal but with cramps and fatigability, 2: an inability to run, 3: walking difficulty but still possible unaided, 4: walking with a cane, 5: walking with crutches, 6 : walking with a walker, and 7: wheelchair bound, and 8, bedridden.

were from 325 (FC290-1) to 434 (FC678-4) with average of 377 from 73 target genes (except for TEKT3). Of them, functionally significant SNVs were observed from 25 (FC182-1) to 45 (FC693-1). These significant SNVs were further selected by the filtering steps of co-segregation analysis within each family, healthy control experiment $(n=300)$, and a screen of global human genome variant databases (dbSNP144, 1000G, ESP, and ExAC).

\section{Identification of causative mutations}

From subsequent filtering and capillary sequencing of candidate variants, we were able to isolate 15 pathogenic or likelypathogenic mutations in 17 families: 6 GJB1 (MIM 304040) mutations in 9 X-linked CMTX1 (MIM 302800) families; 2 MPZ (MIM 159440) mutations in CMT2I (MIM 607677); a pair of compound heterozygote mutations of SH3TC2 (MIM 608206) in a CMT4C (MIM 601596) family; and one mutation each in PMP22 (MIM 601097) in CMT1E (MIM 118300), MARS (MIM 156560) in CMT2U (MIM 616280), MFN2 (MIM 608507) in CMT2A (MIM 609260), SPTLC2 (MIM 605713) in HSAN1C (MIM 613640), and DCTN1 (MIM 601143) in dHMN7B (MIM 607641) (Table 2). Most families showed dominant inheritance of the neuropathy phenotype except a CMT4C family (FC703) with compound heterozygote mutations in SH3TC2.

Eight mutations had not yet been reported as pathogenic mutations. The novel mutations were well co-segregated with affected individual(s) in each family (Fig. 1). The mutations were confirmed by the Sanger's sequencing method (Fig. 2A) and were not found in 300 control samples. In addition, they were not reported or very rarely reported in several global human
A

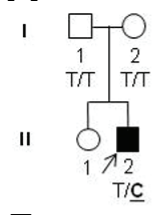

$\mathbf{F}$
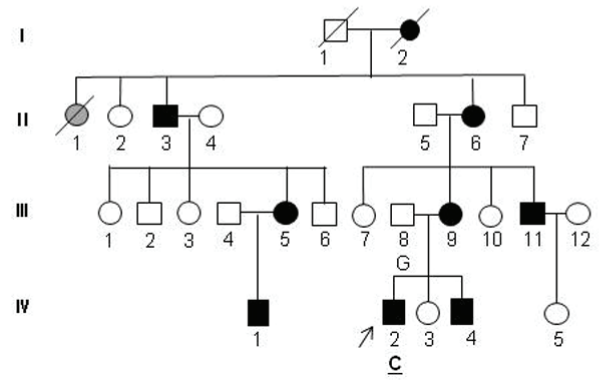

Fig. 1. Pedigrees of inherited peripheral neuropathy families with novel pathogenic or likely pathogenic mutations. Arrows indicate the proband in each family. Open and black symbols represent the unaffected and affected individuals, respectively, whereas grey symbols indicate individuals with no clinical information available. Genotypes of the mutations are indicated at the bottom of all examined individuals (mutant allele: bold underlined): (A) FC541 family with c.47T > C in PMP22, (B) FC703 with c.929G > A and c.3272G > T in SH3TC2, (C) FC156 with c.154T > G in MPZ, (D) FC141 with c.262T > C in MPZ, (E) FC180 with c.1019A > G in DCTN1, (F) FC725 with c.286G >C in GJB1, and (G) FC459 with c.435G > T in SPTLC2. 
Table 3. Characterization of novel pathogenic mutations

\begin{tabular}{|c|c|c|c|c|c|c|c|c|c|}
\hline \multirow{2}{*}{ Genes } & \multirow{2}{*}{ Mutations } & \multicolumn{4}{|c|}{ Human genome DB search ${ }^{a}$} & \multirow{2}{*}{$\mathrm{GERP}^{\mathrm{b}}$} & \multicolumn{3}{|c|}{ In silico analysis ${ }^{\mathrm{C}}$} \\
\hline & & dbSNP144 & $1000 \mathrm{G}$ & ESP & ExAC & & SIFT & PP2 & MUpro \\
\hline PMP22 & p.L16R & - & - & - & - & 3.36 & $0.00^{*}$ & $0.760^{*}$ & $-0.375^{\star}$ \\
\hline \multirow[t]{2}{*}{ SH3TC2 } & p.G310E & rs763949764 & - & - & $<0.001$ & 4.88 & $0.00^{*}$ & $1.000^{*}$ & $-0.288^{*}$ \\
\hline & p.G1091V & rs761592620 & - & - & $<0.001$ & 5.76 & $0.00^{*}$ & $1.000^{*}$ & $-1.000^{\prime}$ \\
\hline \multirow[t]{2}{*}{$M P Z$} & p.F52V & - & - & - & - & 5.29 & $0.00^{*}$ & $1.000^{*}$ & $-0.542^{*}$ \\
\hline & p.Y88H & - & - & - & - & 4.70 & $0.00^{*}$ & $0.997^{*}$ & 0.034 \\
\hline GJB1 & p.A96P & - & - & - & - & 4.67 & $0.00^{*}$ & $1.000^{*}$ & $-0.694^{x}$ \\
\hline SPTLC2 & p.R145S & rs749262868 & - & - & $<0.001$ & 5.23 & $0.01^{*}$ & 0.004 & $-1.000^{\prime}$ \\
\hline DCTN1 & p.E340G & - & - & - & - & 4.32 & $0.00^{*}$ & $1.000^{*}$ & $-0.917^{\star}$ \\
\hline
\end{tabular}

${ }^{2}$ Human genome databases: the GenBank registration No (dbSNP144) or variant allele frequencies at the 1000 Genome Project (1000G: Nov, 2014), the Exome Sequencing Project (ESP: Nov, 2014), and the Exome Aggregation Consortium (ExAC: ver. 0.3)

${ }^{b}$ GERP: genomic evolutionary rate profiling score

${ }^{c}$ In silico analyses using the SIFT (http://sift.jcvi.org), PolyPhen2 (PP2: http://genetics.bwh.harvard.edu/pph2/), and MUpro (http://www.ics.uci.edu/ baldig/mutation) programs

* indicates prediction of pathogenicity.

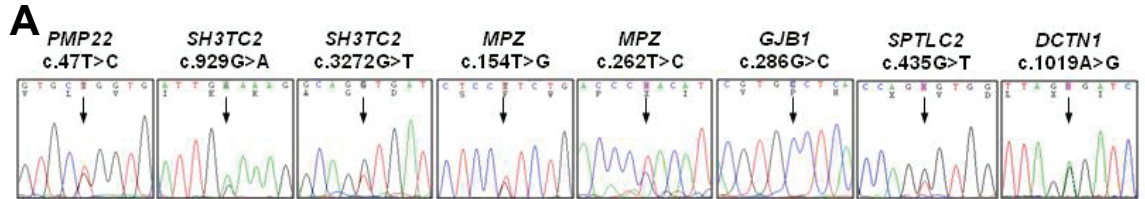

B

\begin{tabular}{|c|c|}
\hline H. sapiens & $H \vee A V L V L L F$ \\
\hline B. taurus & HV A V L V L L F \\
\hline R. norvegicus & H I A V L V L L F \\
\hline M. muscuius & H I A V L V L L \\
\hline G. gallus & $H V T V L V L L F$ \\
\hline \multirow[t]{2}{*}{$X$ laevis } & $H \vee A V L V L L F$ \\
\hline & SH3TC2: G310E \\
\hline H. sapiens & QWF IGKSTS \\
\hline B. taurus & $Q W F I G K S A L$ \\
\hline R. norvegicus & R W F I G K S M S \\
\hline M. musculus & QWF I G K S V S \\
\hline D. rerio & $S M F I G R H L T$ \\
\hline
\end{tabular}

GJB1: A96P

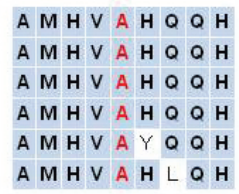

SH3TC2: G1091V

YE E A G D V F F

Y E E A G D V F F

$Y E E A G D V F F$

$Y E E A G D V F F$

YE E A G D V F F

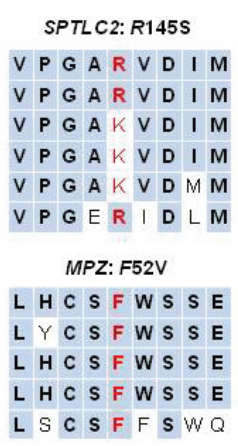

DCTN1: E340G

T T D L E I L K A

T T DLE I L K A

T T D L E I L K A

T T D L E I L K A

T MDLE I L KH

MPZ:Y88H

K G Q P Y I DE V

$K G Q P Y$ I D E V

$K G Q P Y I D E V$

K G Q P Y I D E V

$G G E A Y P A N K$
K I D L E I L K H

Fig. 2. Sequencing chromatograms and conservation analysis of novel causative mutations. (A) Confirmation of the causative mutations using the Sanger's sequencing method. (B) Alignment of the amino acid sequences for the mutation sites and around regions in several vertebrate species: Homo sapiens, Bos taurus, Rattus norvegicus, Mus musculus, Gallus gallus, Xenopus laevis, and Danio rerio.

genome databases, such as dbSNP144, 1000G, ESP, and ExAC (Table 3). The mutation sites were located at the highly conserved regions among the vertebrate species (Fig. 2B). The GERP scores were high at most mutation sites (3.36 to 5.76) and in silico analyses also predicted most of them to be pathogenic. Therefore, we considered these mutations to be the genetic cause.

Interestingly, c.283G > A (p.V95M) mutation in GJB1 was identified in three independent families with a wide range of onset ages (8-48), suggesting a loose genotype-phenotype correlation and mutational hot spot. Mutations in MPZ are implicated into many types of IPN with the most frequent type of demyelinating CMT1B; however, in the present study, both cases with MPZ mutations exhibited less frequent axonal defect of the CMT2I. The p.R280H mutation of MFN2 has shown occasional incomplete penetrance (Choi et al., 2015); however, the present case was completely penetrant within the family (FC527). Two de novo mutations were suggested in FC541 family with p.L16R in PMP22 and FC141 family with p.Y88H in $M P Z$. No same mutation was found in the unaffected parents of FC541 family; whereas de novo event were predicted by the history taking in the FC141 family.

\section{Detection of duplication of chromosome 17p12 region}

We calculated the effectiveness of the gene panel in detecting the duplication of $1.4 \mathrm{Mb}$ in the $17 \mathrm{p} 12$ region. We applied 15 samples of CMT1A duplication patients who were previously confirmed by the microsatellite hexaplex PCR and real-time PCR methods. Read depths of PMP22 and TEKT3 genes, which are located in the duplication region (17p12), were compared with the average read depth of total targets in each targeted sequencing data. Compared with five non-duplicated healthy controls, CMT1A patients showed significantly higher read depths in both genes: $1.496 \pm 0.098$ for PMP22 and $1.472 \pm 0.119$ for TEKT3, with a mean of $1.484 \pm 0.105$ for both genes (Table 4). Because 
Gene Panel for Molecular Diagnosis of IPN

Soo Hyun Nam et al.

Table 4. Detection of copy number variation for chromosome $17 \mathrm{p} 12$ region

\begin{tabular}{|c|c|c|c|}
\hline \multirow{2}{*}{ Samples } & \multicolumn{2}{|c|}{ Read depth ratios ${ }^{a}$} & \multirow{2}{*}{ Mean } \\
\hline & PMP22 & TEKT3 & \\
\hline Controls $(n=5)$ & $1.000 \pm 0.060$ & $1.000 \pm 0.082$ & $1.000 \pm 0.069$ \\
\hline FC425-1 & 1.081 & 1.046 & $1.064 \pm 0.025$ \\
\hline FC453-1 & 0.989 & 0.962 & $0.976 \pm 0.019$ \\
\hline FC565-1 & 1.064 & 1.079 & $1.072 \pm 0.011$ \\
\hline FC703-1 & 0.872 & 0.935 & $0.904 \pm 0.045$ \\
\hline FC707-1 & 0.993 & 0.977 & $0.985 \pm 0.011$ \\
\hline CMT1A patients $(n=15)$ & $1.496 \pm 0.098$ & $1.472 \pm 0.119$ & $1.484 \pm 0.105$ \\
\hline FC045-12 & 1.435 & 1.440 & $1.438 \pm 0.003$ \\
\hline FC144-1 & 1.468 & 1.439 & $1.453 \pm 0.020$ \\
\hline FC168-1 & 1.615 & 1.630 & $1.623 \pm 0.010$ \\
\hline FC175-1 & 1.468 & 1.459 & $1.463 \pm 0.006$ \\
\hline FC179-1 & 1.518 & 1.568 & $1.543 \pm 0.035$ \\
\hline FC214-3 & 1.678 & 1.705 & $1.692 \pm 0.018$ \\
\hline FC215-2 & 1.395 & 1.381 & $1.388 \pm 0.009$ \\
\hline FC226-1 & 1.548 & 1.528 & $1.538 \pm 0.014$ \\
\hline FC287-1 & 1.578 & 1.418 & $1.498 \pm 0.113$ \\
\hline FC339-1 & 1.623 & 1.649 & $1.636 \pm 0.018$ \\
\hline FC498-1 & 1.328 & 1.303 & $1.315 \pm 0.017$ \\
\hline FC511-1 & 1.414 & 1.348 & $1.381 \pm 0.047$ \\
\hline FC512-1 & 1.414 & 1.422 & $1.418 \pm 0.005$ \\
\hline FC561-1 & 1.504 & 1.436 & $1.470 \pm 0.048$ \\
\hline FC589-1 & 1.449 & 1.356 & $1.402 \pm 0.065$ \\
\hline
\end{tabular}

${ }^{\mathrm{a}}$ Read depth ratios were determined by comparison with the mean read depth of control samples ( $\left.\mathrm{n}=5\right)$ in each PMP22 and TEKT3 gene (control was indicated by 1.000).

these values are approaching the hypothetical value of 1.5fold, the gene panel seems to be sufficient to detect the 1.4 $\mathrm{Mb}$ duplication of CMT1A patients.

\section{Clinical features of IPN patients}

The brief clinical manifestations of affected individuals are shown in Table 2. The onset ages ranged from 1 to 49 years for the proband individuals, where the ages were earlier than 5 years in patients with PMP22, SH3TC2 and MARS mutations. High FDS (score 6 or 7) was found only in the FC541 with PMP22 mutation, the other patients showed mild or moderate symptoms with low FDSs (score $\leq 3$ ). This targeted gene panel study, in particular, found several very rare mutations in SPTLC2, DCTN1, and MARS.

A novel p.R145S mutation in SPTLC2 was identified in a HSAN1C female patient (FC395) with onset of second decade. She revealed progressive sensory impairment resulting in ulcerations and amputations. Sensory loss occurred in a glove and stocking distribution, and pinprick perception was affected to a greater extent than vibration. She had painful tingling in both hands. Sensory complications including ulcers and accidental burns occurred, and severe wasting and weakness were present. SPTLC2 is a subunit of serine palmitoyltransferase (SPT) enzyme, and a crucial element in the de novo biosynthesis of sphingolipids. To date, only several HSAN1C families have been described (Ernst et al., 2015; Rotthier et al., 2010), and this was the first reported Asian case.

A 12-year-old boy (FC180) showed a novel p.E340G mutation in DCTN1, which is known to cause dHMN7B. He com- plained of gait difficulty and hand muscle weakness at the age of 10 years. He had respiratory distress while talking, and distal dominant weakness in upper and lower limbs. In his hands, there was atrophy and weakness of the first dorsal interosseous and thenar muscles, but his hypothenar muscle bulk and strength was relatively preserved. Sensation was intact to all modalities. The findings of nerve conduction study and electromyogram were consistent with pure motor neuropathy. The DCTN1 encodes the largest subunit of dynactin 1, which plays an important role in retrograde axonal transport of vesicles and organelles. Since the first dHMN7B patient were described in 2003 (Puls et al., 2003), there has not been another case reported.

This study also found a CMT2U family (FC495) with p.P800T mutation in MARS, which was previously reported in a lateonset (50-60 years of age) CMT2 family (Hyun et al., 2014). However, the present family with the same MARS mutation showed relatively early onset development (ages of 5 and 16 years) compared to previous case. This suggests variability of age at onset in CMT2U. Until now, only two MARS mutations have been reported to be associated with axonal CMT (Gonzalez et al., 2013; Hyun et al., 2014).

\section{DISCUSSION}

We developed a new IPN diagnostic tool that enables one-step detection of both 17p12 duplication and SNVs in target genes. Traditionally, genetic diagnosis of IPN adopted a tiered detection system, with considerable time and cost, involves determination of phenotype based on motor nerve conduction velocity, 
detection of chromosome $17 \mathrm{p} 12$ region duplication; then capillary sequencing for major genes with high frequency (Murphy et al., 2013; Saporta et al., 2011). In accordance with the technological ripeness in NGS, the simplest diagnostic algorithm was also proposed, where exome sequencing was included right after screening of PMP22 duplication for CMT1, and MFN2 mutations for CMT2 (Chung et al., 2006). In spite of the laborious procedures in each step, however, the rate of successful isolation has been less than $60 \%$.

Compared to previous processes, the newly developed diagnostic system strikingly reduced the time and cost with better detection efficiency. We applied the new system to 74 nonCMT1A patients (63 families) and isolated the causative mutations from 25 patients (17 families). Considering that CMT1A families were ruled out in this study, the gene panel exhibited high diagnostic yield. Technically, the major difference between WES and the targeted gene panel is the number of genes, which determines the cost and time. In addition, the reduced number of target sequences enhances read-depth and coverage, the major concerns of WES. Lower read-depth and coverage cause miscalling of false-positives and true-negatives, respectively, of potent SNPs. Using the gene panel, we could increase the read-depth to more than 10 times higher and the coverage to almost $100 \%$. Therefore, application of the new targeted gene panel for IPNs can significantly enhance the effectiveness of genetic diagnosis.

To create a one-step diagnosis system, we also included a detection strategy for the copy number variation (CNV) of the chromosome 17 p12 region. The current method, a short nucleotide read-based NGS, has a disadvantage in the detection of CNV for a long ranged targets. Previously, we demonstrated that detection of PMP22 duplication is simply achieved by the application of six markers with $99.9 \%$ of efficiency (Choi et al., 2007). In the new gene panel system, we designed a CNV detection strategy by comparing the read-depth of marker sequences. Because the mean read-depth of the duplication region was close to 1.5 fold higher than other regions and controls, the new strategy was sufficiently effective for CNV detection of CMT1A. These data also imply that the new gene panel can be applied to detection of HNPP, a disease caused by deletion of the same region.

In addition, the application of this gene panel could be used for association study. Recently, a SNP of LITAF (relating with CMT1C), was reported to be associated with the onset age of CMT1A and HNPP (Sinkiewicz-Darol et al., 2015). Since all IPN causative genes are simultaneously sequenced at the same time, future accumulation of genetic diagnosis data might facilitate the finding of new phenotype-modulating SNPs.

Collectively, we prepared a new one-step diagnosis tool that works without any prescreening, of which applicability was validated. Because the list of genetic causes of IPNs is still growing, continuous updating of the list in the gene panel could facilitate the exact genetic diagnosis of various peripheral neuropathies.

Note: Supplementary information is available on the Molecules and Cells website (www.molcells.org).

\section{ACKNOWLEDGMENTS}

This study was supported by the Korean Health Technology R\&D Project, Ministry of Health \& Welfare (HI12C0135 and HI14C3484), and by the National Research Foundation funded by MSIP (NRF-2014R1A2A2A01004240 and 2014R1A2A2A 01003164), Republic of Korea.

\section{REFERENCES}

Antoniadi, T., Buxton, C., Dennis, G., Forrester, N., Smith, D., Lunt P., and Burton-Jones, S. (2015). Application of targeted multigene panel testing for the diagnosis of inherited peripheral neuropathy provides a high diagnostic yield with unexpected phenotype-genotype variability. BMC Med. Genet. 16, 84.

Bone, L.J., Deschênes, S.M., Balice-Gordon, R.J., Fischbeck, K.H., and Scherer, S.S. (1997). Connexin32 and X-linked CharcotMarie-Tooth disease. Neurobiol. Dis. 4, 221-230.

Choi, B.O., Kim, J., Lee, K.L., Yu, J.S., Hwang, J.H., and Chung, K.W. (2007). Rapid diagnosis of CMT1A duplications and HNPP deletions by multiplex microsatellite PCR. Mol. Cells 23, 39-48.

Choi, B.O., Koo, S.K., Park, M.H., Rhee, H., Yang, S.J., Choi, K.G. Jung, S.C., Kim, H.S., Hyun, Y.S., Nakhro, K., et al. (2012). Exome sequencing is an efficient tool for genetic screening of Charcot-Marie-Tooth disease. Hum. Mutat. 33, 1610-1615.

Choi, B.O., Nakhro, K., Park, H.J., Hyun, Y.S., Lee, J.H., Kanwal, S. Jung, S.C., and Chung, K.W. (2015). A cohort study of MFN2 mutations and phenotypic spectrums in Charcot-Marie-Tooth disease 2A patients. Clin. Genet. 87, 594-598.

Chung, K.W., Kim, S.B., Park, K.D., Choi, K.G., Lee, J.H., Eun, H.W., Suh, J.S., Hwang, J.H., Kim, W.K., Seo, B.C., et al. (2006) Early onset severe and late-onset mild Charcot-Marie-Tooth disease with mitofusin 2 (MFN2) mutations. Brain 129, 2103-2118.

Drew, A.P., Zhu, D., Kidambi, A., Ly, C., Tey, S., Brewer, M.H., Ahmad-Annuar, A., Nicholson, G.A., and Kennerson, M.L. (2015) Improved inherited peripheral neuropathy genetic diagnosis by whole-exome sequencing. Mol. Genet. Genomic Med. 3, 143154.

Ernst, D., Murphy, S.M., Sathiyanadan, K., Wei, Y., Othman, A. Laurá, M., Liu, Y.T., Penno, A., Blake, J., Donaghy, M., et al. (2015). Novel HSAN1 mutation in serine palmitoyltransferase resides at a putative phosphorylation site that is involved in regulating substrate specificity. Neuromolecular Med. 17, 47-57.

Gonzaga-Jauregui, C., Harel, T., Gambin, T., Kousi, M., Griffin, L.B., Francescatto, L., Ozes, B., Karaca, E., Jhangiani, S.N., Bainbridge, M.N., et al. (2015). Exome sequence analysis suggests that genetic burden contributes to phenotypic variability and complex neuropathy. Cell Rep. 12, 1169-1183

Gonzalez, M., McLaughlin, H., Houlden, H. Guo, M., Yo-Tsen, L., Hadjivassilious, M., Speziani, F., Yang, X.L., Antonellis, A., Reilly, M.M., et al. (2013). Exome sequencing identifies a significant variant in methionyl-tRNA synthetase (MARS) in a family with late-onset CMT2. J. Neurol. Neurosurg. Psychiatry 84, 12471249.

Hyun, Y.S., Park, H.J., Heo, S.H., Yoon, B.R., Nam, S.H., Kim, S.B., Park, C.I., Choi, B.O., and Chung, K.W. (2014). Rare variants in methionyl- and tyrosyl-tRNA synthetase genes in late-onset autosomal dominant Charcot-Marie-Tooth neuropathy. Clin. Genet. 86, 592-594.

Lupski, J.R., and Garcia, C.A. (2000). Charcot-Marie-Tooth peripheral neuropathies and related disorders. In the metabolic and molecular bases of inherited disease. Vol. 4. $8^{\text {th }}$, C.R. Scriver, ed. (New York, Mc Graw Hill), pp. 5759-5788.

Montenegro, G., Powell, E., Huang, J. Speziani, F., Edwards, Y.J., Beecham, G., Hulme, W., Siskind, C., Vance, J., Shy, M., et al. (2011). Exome sequencing allows for rapid gene identification in a Charcot-Marie-Tooth family. Ann. Neurol. 69, 464-470.

Murphy, S.M., Laurá, M., and Reilly, M.M. (2013). DNA testing in hereditary neuropathies. Handb. Clin. Neurol. 115, 213-232.

Nakhro, K., Park, J.M., Choi, B.O., and Chung, K.W. (2013). Missense mutations of mitofusin 2 in axonal Charcot-Marie-Tooth neuropathy: polymorphic or incomplete penetration?. Anim. Cells Syst. 17, 228-236.

Nelis, E., Van Broeckhoven, C., De Jonghe, P., Löfgren, A., Vandenberghe, A., Latour, P., Le Guern, E., Brice, A., Mostacciuolo, M.L., Schiavon, F., et al. (1996). Estimation of the mutation frequencies in Charcot-Marie-Tooth disease type 1 and hereditary neuropathy with liability to pressure palsies: a European collaborative study. Eur. J. Hum. Genet. 4, 25-33.

Oterino, A., Montón, F.I., Cabrera, V.M., Pinto, F., Gonzalez, A., and Lavilla, N.R. (1996). Arginine-164-tryptophan substitution in connexin32 associated with $\mathrm{X}$ linked dominant Charcot-MarieTooth disease. J. Med. Genet. 33, 413-415. 
Patzkó, A., and Shy, M.E. (2011). Update on Charcot-Marie-Tooth disease. Curr. Neurol. Neurosci. Rep. 11, 78-88.

Puls, I., Jonnakuty, C., LaMonte, B.H., Holzbaur, E.L., Tokito, M., Mann, E., Floeter, M.K., Bidus, K., Drayna, D., Oh, S.J., et al. (2003). Mutant dynactin in motor neuron disease. Nat. Genet. 33, 455-456.

Rossor, A.M., Polke, J.M., Houlden, H., and Reilly, M.M. (2013). Clinical implications of genetic advances in Charcot-Marie-Tooth disease. Nat. Rev. Neurol. 9, 562-571.

Rotthier, A., Auer-Grumbach, M., Janssens, K., Baets, J., Penno, A., Almeida-Souza, L., Van Hoof, K., Jacobs, A., De Vriendt, E., Schlotter-Weigel, B., et al. (2010). Mutations in the SPTLC2 subunit of serine palmitoyltransferase cause hereditary sensory and autonomic neuropathy type I. Am. J. Hum. Genet. 87, 513-522.

Rudnik-Schöneborn, S., Tölle, D., Senderek, J., Eggermann, K., Elbracht, M., Kornak, U., von der Hagen, M., Kirschner, J., Leube, B., Müller-Felber, W., et al. (2016). Diagnostic algorithms in Charcot-Marie-Tooth neuropathies: experiences from a German genetic laboratory on the basis of 1206 index patients. Clin.
Genet. 89, 34-43.

Saporta, A.S., Sottile, S.L., Miller, L.J., Feely, S.M., Siskind, C.E., and Shy, M.E. (2011). Charcot-Marie-Tooth disease subtypes and genetic testing strategies. Ann. Neurol. 69, 22-33.

Schiavon, F., Fracasso, C., and Mostacciuolo, M.L. (1996). Novel missense mutation of the connexin32 (GJB1) gene in X-linked dominant Charcot-Marie-Tooth neuropathy. Hum. Mutat. 8, 8384.

Sinkiewicz-Darol, E., Lacerda, A.F., Kostera-Pruszczyk, A., Potulska-Chromik, A., Sokołowska, B., Kabzińska, D., Brunetti, C.R., Hausmanowa-Petrusewicz, I., and Kochański, A. (2015). The LITAF/SIMPLE I92V sequence variant results in an earlier age of onset of CMT1A/HNPP diseases. Neurogenetics 16, 27-32.

Ylikallio, E., Johari, M., Konovalova, S., Moilanen, J.S., Kiuru-Enari, S., Auranen, M., Pajunen, L., and Tyynismaa, H. (2014). Targeted next-generation sequencing reveals further genetic heterogeneity in axonal Charcot-Marie-Tooth neuropathy and a mutation in HSPB1. Eur. J. Hum. Genet. 22, 522-527. 